\title{
Advanced methods for space-time environmental data
}

\author{
Alessandro Fassò $^{1}$ • Abdel El-Shaarawi ${ }^{2}$. \\ Maria Giovanna Ranalli ${ }^{3}$
}

Accepted: 30 November 2015 / Published online: 23 December 2015

(C) Springer-Verlag Berlin Heidelberg 2015

The METMA-GRASPA joint meeting held in Turin, 10-12 September, 2014, brought together two communities with the common research interest for the development and use of statistical methods in the environmental sciences and for these reasons it was also a regional conference of the International environmetrics society (www. environmetrics.org).

It was the first international conference organized together by GRASPA, which is the permanent working group for environmental statistics of the Italian statistical society, and METMA, at its seventh conference. During the meeting, considering not only the number and scientific level of the contributions, but also the general interest of the topic, which exceeds the conference coverage, the idea of a special issue on "advanced methods for space-time environmental data" arose in a natural way.

As guest editors we are really thankful to the past SMAP's editor Andrea Cerioli and co-editor Lucio Barabesi for their continuous help and advice. We also appreciated very much the scientific level of all the contributions and we recognize with gratitude the effort and scientific competence of all the anonymous referees involved in the review process. Moreover, we express our sincere thanks to the editorial staff and the managing editor Francesca Torti.

\footnotetext{
Alessandro Fassò

alessandro.fasso@unibg.it

Abdel El-Shaarawi

elshaarawi@feps.edu.eg

Maria Giovanna Ranalli giovanna@stat.unipg.it

1 University of Bergamo, Bergamo, Italy

2 Cairo University, Giza, Egypt

3 University of Perugia, Perugia, Italy
} 
The result is an interesting mix of applied and theoretical papers, covering important environmental problems and interesting statistical issues. Ordering applications from global to local, we have: global carbon budget using space-time hierarchical models, geostatistical space-time trend analysis of air quality in Portugal, wind speed forecast at regional level and river temperature trends under irregular sampling.

Methodological papers give new insight into optimal sampling for spatial functional data, nonparametric regression for spatial compositional data, skew normal spatial models, covariance tapering of large multivariate spatial datasets, and, last but not least, new compactly supported space-time covariance functions.

In more details, Vetter et al. (2015) develop a space-time statistical model which is interesting to statisticians, ecologists and environmental economists. Indeed, an empirical quantitative explanation of the global space-time variation of the Net Ecosystem Exchange in terms of sink effect/source effect of $\mathrm{CO} 2$ is searched using a sizeable global dataset with state-of-the-art hierarchical modeling. In particular the Net Ecosystem Exchange is inferred from remotely-sensed carbon dioxide ground concentrations together with data on the Normalized Difference Vegetation Index, the Gross Primary Production and the land cover classification. The model is based on spatial and temporal latent random effects, that act as space-time varying coefficients. Despite the high noise to signal ratio the authors find meaningful new results.

Menezes et al. (2015) propose a geostatistical analysis of space-time trends of nitrogen dioxide $\left(\mathrm{NO}_{2}\right)$ using 9 years of daily data from the national network in Portugal. They propose a two stage estimation techniques, first they fit a deterministic trend for space-time effects using a Gamma GLM model and then, they fit a space-time geostatistical model to the residuals. In order to do correct inference on trend coefficients these authors compute standard deviations of the estimates using bootstrap and show the difference with the over optimistic standard deviations obtained by the incorrectly specified model used in the first stage.

Ambach and Croonenbroeck (2015), in the frame of wind power, discuss modeling of wind speed for high frequency time series data coming from a limited number of spatially distributed wind stations. Since the paper objective is short- to medium-term temporal in situ forecast, they try to get the maximum of the complex space-time dynamics using a semiparametric non stationary vector autoregressive model with an asymmetric periodic heteroskedastic component. Here the multivariate response includes wind and other meteorological variables from the seven meteorological stations. Due to the large number of candidate parameters arising from the lagged components, they use lasso and elastic net estimation to estimate the B-spline autoregressive parameters for both mean and variance.

Gray et al. (2015) consider statistical modelling along rivers and streams with an application to temperature trend analysis of Illinois river in USA. It is observed that monitoring of water bodies is often based on irregular sampling techniques which are irregular in space and time. Moreover, using Monte Carlo simulations, they show that trends in sampling strategy entail bias on trend estimates. To solve this problem they propose a multilevel model including random and fixed effects for year, day, time and longitude.

Bohhorquez et al. (2015) propose optimal sampling formulas for spatial functional data which are becoming more and more important as far as geostatistics of complex 
objects is developing in various application fields. For example, in climate statistics, spatial functional data naturally arise when observations are in the form of atmospheric profiles coming e.g. from radiosonde on balloon flights or satellite optical devices, collected around the globe, hence spatially indexed. See for example Fassò et al. (2014).

Nonparametric regression for a compositional covariate coming from spatially correlated data is considered by Bruno et al. (2015). The approach is based on isometric log-ratios of the compositional part and a smooth function over the resulting multidimensional space. Smoothness is achieved by Bayesian P-splines with random walk priors. The proposed methodology is applied to gridded data from an ecological survey on a gypsum outcrop located in Bologna province, Italy.

In the frame of non Gaussian random fields, Boojari et al. (2015) develop a fixed rank kriging model, assuming that the non-spatial latent variables have a skew-normal distribution. Adopting a Bayesian framework, MCMC algorithm is easily implemented using BUGS package, giving parameter estimates and kriging interpolation. This paper has some relations to above mentioned paper of Menezes et al. (2015) which also has asymmetric distributed response variables. However, the latter is a space-time model with two stage estimation, while Boojari et al. do not consider time and use a hierarchical model instead.

The last two papers consider theoretical results regarding space and space-time covariance models with compact support. In particular, Bevilacqua et al. (2015) consider estimation of the spatial covariance structure for a multivariate Gaussian random field using large datasets. To do this, they work with the so called two-taper tapering, which leads to unbiased estimation equations. Using a large simulation campaign the asymptotic behavior under increasing domain is studied for a battery of separable and non-separable tapers. The authors conclude that, when the focus is on the estimation of the parameters associated to specific marginal components of the multivariate random fields, the use of a non-separable taper is recommended.

Last but not least, Ruiz-Medina et al. (2015) present a new class of fractional spacetime covariance models giving Gaussian random fields characterized by compactly supported non-separable space-time covariance kernels admitting a parametric representation. The associated local fractional exponents are estimated from the empirical log-wavelet variogram.

\section{References}

Ambach D, Croonenbroeck C (2015) Space-time short- to medium-term wind speed forecasting. Stat Methods Appl. doi:10.1007/s10260-015-0343-6

Bevilacqua M, Fassò A, Gaetan C, Porcu E, Velandia D (2015) Covariance tapering for multivariate Gaussian random fields estimation. Stat Methods Appl. doi:10.1007/s10260-015-0338-3

Bohhorquez M, Giraldo R, Mateu J (2015) Optimal sampling for spatial prediction of functional data. Stat Methods Appl. doi:10.1007/s10260-015-0340-9

Boojari H, Khaledi MJ, Rivaz F (2015) A non-homogeneous skew-Gaussian Bayesian spatial model. Stat Methods Appl. doi:10.1007/s10260-015-0331-X

Bruno F, Fedele G, Ventrucci M (2015) Non-parametric regression on compositional covariates using Bayesian P-splines. Stat Methods Appl. doi:10.1007/s10260-015-0339-2

Fassò A, Ignaccolo R, Madonna F, Demoz B, Franco-Villoria M (2014) Statistical modelling of collocation uncertainty in atmospheric thermodynamic profiles. Atmos Meas Tech 7:1803-1816 
Gray BR, Lyubchich V, Gel YR, Rogala JT, Robertson DM, Wei X (2015) Estimation of river and stream temperature trends under haphazard sampling. Stat Methods Appl. doi:10.1007/s10260-015-0334-7

Menezes Piairo H, Garcia-Soidan P, Sousa I (2015) Spatial-temporal modellization of the $\mathrm{NO}_{2}$ concentration data through geostatistical tools. Stat Methods Appl. doi:10.1007/s10260-015-0346-3

Ruiz-Medina MD, Angulo JM, Christakos G, Fernández-Pascual R (2015) New compactly supported spatiotemporal covariance functions from SPDEs. Stat Methods Appl. doi:10.1007/s10260-015-0333-8

Vetter P, Schmid W, Schwarze R (2015) Spatio-temporal statistical analysis of the carbon budget of the terrestrial ecosystem. Stat Methods Appl. doi:10.1007/s10260-015-0342-7 